\title{
LA DISSEMINAZIONE DELLA MUSICA DI GIUSEPPE TARTINI IN FRANCIA LE EDIZIONI SETTECENTESCHE DI SONATE PER VIOLINO E BASSO
}

\author{
CANDIDA FELICI \\ Conservatorio di Musica di Cosenza
}

\begin{abstract}
Izvleček: Prispevek obravnava francoske izdaje sonat za violino in basso continuo Giuseppa Tartinija. Podatki iz najav pariškega časopisja in založniških katalogov so omogočili datacijo izdaj skladateljevih opusov 4 do 9 vetih 1747-1750. Primerjava z rokopisnimi viri Tartinijevih sonat nadalje omogoča oceno pomena francoskih izdaj, med katerimi so bile sonate iz op. 6 in 7 največkrat prepisane. O zvezah Tartinija s francoskimi založniki se žal ne ve nič.
\end{abstract}

Ključne besede: Giuseppe Tartini,violinske sonate, francoske izdaje, André Noël Pagin.

\begin{abstract}
This article examines eighteenth-century French editions of sonatas for violin and bass by Giuseppe Tartini. Through the announcements in the Parisian journals and the catalogues of music publishers, it has been possible to establish the years of issue for op. 4 to op. 9 to the period 1747-1750. The comparison with the manuscript sources of Tartini sonatas allows to evaluate the reliability of these editions, of whom op. 6 and 7 result those with greater number of concordances, even if we have no knowledge of relations between Tartini and the French publishers.
\end{abstract}

Keywords: Giuseppe Tartini, violin sonatas, French editions, André Noël Pagin.

Le edizioni di sonate di Giuseppe Tartini apparse durante la vita del compositore sono più numerose di quelle dei concerti, la maggior parte dei quali ci è pervenuta attraverso fonti manoscritte. Tuttavia sappiamo che Tartini autorizzò soltanto due stampe di sonate per violino e basso continuo, l'edizione op. 1 apparsa ad Amsterdam presso Le Cène nel $1734^{1}$ e l'op. 2 stampata a Roma da Cleton nel $1745 .{ }^{2}$ Riguardo le altre edizioni apparse in Olanda, Inghilterra e Francia, Tartini non ne seppe nulla, o, quando ne ebbe notizia, lo seppe a cose già fatte, senza alcun controllo sulle composizioni selezionate per la stampa

* Nel presente articolo sono usate le seguenti abbreviazioni:

A-Wgm Wien, Archiv der Gesellschaft der Musikfreunde

I-Pca Padova, Biblioteca Antoniana

F-Pc Paris, Bibliothèque Nationale, Fonds du Conservatoire

S-Skma Stockholm, Musik- och teaterbiblioteket

US-BEm Berkeley, University of California, Music Library

1 Tartini, Sonate a Violino e Violoncello, 1734.

2 Tartini, Sonate a Violino e Basso, 1745. 
o sulla loro correttezza testuale, e senza averne alcun vantaggio economico, come alcune sue lettere ci testimoniano:

Poi le dico che, nulla sapendo io se non confusamente delle mie suonate uscite nuovamente alla stampa, ho procurato per mezo del signor console di Olanda, ch'è in Venezia, di saperne l'intiero. È verissimo dunque che sono uscite alla luce sei mie suonate a solo, ma è altrettanto vero, che mi è riuscito improviso questo fatto, e che io non ho interesse alcuno con lo stampator delle mede(si)me, ch'è Olandese. Non le ho dunque ne men io, e se lo stampatore non manda esemplari in Italia per vendita, non le avrò mai. Se poi li manda, dovrò anch'io comprare la robba mia. Mà intanto V(ostra) S(ignoria) Ill(ustrissi) ma che può aver mezi dove e come vuole, mi farebbe una somma grazia, se procurasse di farsi venire Sei esemplari delle medeme dà Amsterdam, dove sono stampate per gli Eredi di Michiel Charle le Cene. ${ }^{3}$

D’altronde, attraverso i numerosi allievi provenienti da ogni angolo d'Europa, molte composizioni ed opere teoriche tartiniane presero il largo, malgrado alcune lettere del compositore ci testimonino l'attenzione volta ad evitare che le composizioni venissero copiate senza permesso; tuttavia ciò contrastava con l'attività didattica, in cui un aspetto rilevante era costituito proprio dall'imitazione stilistica che comportava anche trascrivere le composizioni del maestro, magari dotandole di una nuova veste ornamentale.

Si può affermare che sia nel caso dell'op. 1 sia in quello dell'op. 2 la scelta di pubblicare le sonate fu determinata dal desiderio di contrastare le stampe pirata:

[Non le ho risposto perché] sinora sono stato e sono attualmente occupato nello scrivere per mettere in stampa dodeci sonate a solo [op. 1 Le Cène], non per mia volontà, ma forzato da una cattiva azione fattami da uno stampatore olandese. ${ }^{4}$

Dunque, la stampa delle dodici sonate che apparve presso Le Cène nel 1734, dedicata a Girolamo Ascanio Giustiniani, fu in qualche modo una reazione all'edizione Witvogel delle sonate ${ }^{5}$ - op. 1 - o all'edizione Le Cène dei concerti - Libro primo, volumi 1, 2, 36 - stampati negli anni 1727 e $1729 .{ }^{7}$ Se Tartini nella sua lettera del marzo 1731 si riferiva all'edizione delle sonate, come pare probabile, questa dovrebbe essere datata al 1730-1731 e non al 1732 come riportato da Paul Brainard. ${ }^{8}$ Si rivelano inoltre

3 Lettera di Giuseppe Tartini a Giuseppe Valeriano Vannetti, 19 marzo 1744. Il testo delle lettere citate nel presente saggio, tratto dalle trascrizioni realizzate da Pierluigi Petrobelli, è stato gentilmente messo a mia disposizione da Sergio Durante. Sulla problematica relativa al rapporto tra Tartini e gli editori cfr. Durante, "Tartini and his texts," 167-207: 180-185.

4 Lettera di Giuseppe Tartini a padre Giovanni Battista Martini, 31 marzo 1731.

5 Tartini, VI Sonate a Violino e Violone.

6 Tartini, Sei Concerti a Cinque è Sei Stromenti [... .] Opera Prima Libro Primo; Id. Sei Concerti a Cinque Stromenti Opera Prima Libro Secondo; Id., Sei Concerti a Cinque Stromenti [... ] Opera Prima Libro Terzo.

7 Sui concerti di Tartini cfr. Canale Degrassi, "I concerti solistici di Giuseppe Tartini." Per la datazione delle stampe di Le Cène cfr. ivi, vol. 1, 54-55 e Lesure, Bibliographie, 53; cfr. inoltre Rasch, The Music Publishing House, Catalogue Taglietti-Trios, 8-11.

8 Cfr. Brainard, Le sonate per violino, xxxv. 
interessanti le parole dello stampatore La Coste,${ }^{9}$ il quale rilevò la casa editrice dagli eredi di Le Cène - morto nel 1743 - e al quale Tartini si rivolge attraverso la persona di Giuseppe Valeriano Vannetti di Rovereto per proporgli l'acquisto di alcune copie dell'edizione romana op. 2: l'accordo proposto da Tartini non viene accolto da La Coste, che pretenderebbe condizioni a lui più favorevoli; tuttavia lo stampatore nella sua missiva fa riferimento ad un'edizione olandese precedente l'op. 1 Le Cène:

j'ay une grande admiration pour ce celebre auteur, mon predecesseur a eu de luy plusieurs manuscrits de concerto et de Solo, qui sont gravez, et qui suffisent pour faire son eloge. il y a icy un homme qui en a fait graver sous son nom, mais le public n'en a pas eté sa dupe, et a bien reconnu la difference qu'il y a de cès ouvrages à ces premiers. ${ }^{10}$

A chi La Coste si riferisca non è dato al momento sapere; tuttavia la lettera ci mostra la fragilità della posizione del compositore e la facilità con cui potevano essergli attribuite composizioni non sue. In effetti, se si esamina il contenuto della prima edizione di sonate tartiniane - op. 1 Witvogel - si vede che solo due di esse (A3 e B8) ${ }^{11}$ appaiono in numerose fonti manoscritte non derivate da stampe, mentre la a9 non compare in alcun manoscritto, la h5 e la g9 concordano con fonti manoscritte probabilmente derivate dalla stessa stampa Witvogel e la B7 concorda con la sonata n. 2 dell'op. 7, che ha però un primo movimento diverso (si veda la Tavola 2). Sembrerebbe possibile che Witvogel non avesse a disposizione un numero di sonate tartiniane tali da poter mettere insieme il consueto gruppo di sei e che quindi abbia fatto ricorso a composizioni di altri autori; le parole di La Coste potrebbero avvalorare questa ipotesi e spiegare anche la forte preoccupazione di Tartini per la "cattiva azione fattami da uno stampatore olandese". ${ }^{2}$

Malgrado Le Cène avesse pubblicato l'op. 1 delle sonate con il consenso dell'autore, non si peritò di ottenerlo per la stampa dell'op. 2 (1743), ${ }^{13}$ come la lettera su citata del 19 marzo 1744 chiarisce.

Se i riferimenti alle stampe olandesi delle sonate, autorizzate o meno, non mancano nelle lettere di Tartini, non vi troviamo invece alcun richiamo alle edizioni francesi. Queste ultime, a partire dagli anni Quaranta, si occupano al principio di rieditare le stampe di Le Cène e Cleton (op. 1 e op. 2 Le Cène e op. 2 Cleton, quest'ultima ristampata a Parigi come op. 3), per poi pubblicare dall'op. 4 all'op. 9 sonate almeno in parte inedite. Queste raccolte uscirono per i tipi di Le Clerc, Hue e Maupetit. ${ }^{14}$ Ad esse si deve aggiungere

9 Cfr. Rasch, "Publishers and Publishers," 194-195; dopo la morte di Le Cène nel 1743, EmanuelJean de la Coste acquistò l'impresa e continuò l'attività fino al 1746.

${ }^{10}$ Lettera di Emanuel-Jean de la Coste a Giuseppe Valeriano Vannetti, 8 dicembre 1744.

${ }^{11}$ Le sigle corrispondono a quelle del catalogo Brainard.

${ }^{12}$ Vedi sopra nota 4.

${ }^{13}$ Tartini, VI Sonate a Violino e Violoncello, [1743].

${ }^{14}$ Sull'editoria musicale francese nel Settecento cfr. Devriès-Lesure, Edition et commerce de la musique; Id., "Paris et la dissémination d'éditions musicales." Sui frontespizi delle edizioni francesi erano indicati, oltre allo stampatore (con l'allocuzione "gravé par"), l'editore e i librai (spesso essi stessi editori) presso cui era possibile acquistare i volumi. Malgrado non sia sempre facile distinguere nel frontespizio il ruolo dell'uno e degli altri, l'editore (il quale può anche coincidere con lo stampatore) si desume sia dal catalogo spesso accluso all'edizione, sia dal 
L'arte dell'arco, ovvero le variazioni sulla Gavotta della sonata op. 5 n. 10 di Corelli, apparse dapprima per i tipi di Hue (prima del o nel 1747),${ }^{15}$ comprendenti 17 variazioni, e poi pubblicate da Le Clerc con 38 variazioni; infine L'Art du Violon, ${ }^{16}$ antologia di Jean Baptiste Cartier del 1798 che comprende, oltre alle sonate n. 1 dell'op. 1 e op. 2 Le Cène (A14, g4), L’Art de l'archet (stavolta con 50 variazioni), la famosa Sonate de Tartini, que son école avoit nommée le Trille du Diable (g5) ${ }^{17}$ e 17 variazioni sul primo tempo, Adagio, della sonata F5.

Oltre alle raccolte di sonate la disseminazione della musica di Tartini in Francia passò anche attraverso la trascrizione di sonate per altro organico, ${ }^{18}$ la pubblicazione del Traité des agrémens de la musique ${ }^{19}$ a Parigi nel 1771, a un anno dalla morte del compositore, senza che l'autore ne avesse mai approntato una versione per la stampa, e la Lettera all'allieva veneziana Maddalena Lombardini, ${ }^{20}$ che, apparsa a Venezia nel giornale L'Europa letteraria nel 1770, fu edita in traduzione francese nel Journal de Musique del 1773.

Si potrebbe ipotizzare che le stampe francesi di sonate a violino e basso siano da collegarsi al violinista e compositore André Noël Pagin, allievo di Tartini assai apprezzato al Concert Spirituel proprio negli anni 1747-1750, come si può leggere nel Mercure de France:

- 8 dicembre 1747, "M. Pagin Symphoniste habile, exécuta une Sonate à violon seul, de sa composition, qui fut applaudie"; 21

- 2 febbraio 1748, festa della Candelora, Pagin suona da solo "au gré des connoisseurs"; 22

- novembre 1748, festa di tutti I Santi, "M. Pagin joüa un Concerto du fameux

Tartini, \& selon sa coûtume, il charma par la finesse de son exécution les oreilles connoisseuses"; 23

- 15 maggio 1749, festa dell'Ascensione, nello stesso concerto in cui veniva eseguita una sinfonia di Geminiani "M. Lavaux a joué sur le haut-bois un Duo avec M. Pagin"

privilegio reale talvolta presente alla fine del volume, che consentiva la stampa in esclusiva dell'opera in questione.

15 Tartini, Nouvelle étude. Dal catalogo delle opere stampate da Hue, accluso all'edizione dell'op. 4, risulta già pubblicata presso lo stesso stampatore la Nouvelle étude, quindi, poiché l'op. 4 è databile presumibilmente al 1747, le variazioni sulla Gavotta di Corelli devono essere state pubblicate prima o durante il 1747.

${ }^{16}$ Cartier, L'art du violon. Sulla disseminazione della musica di Tartini in Francia e in particolare su L'Art du violon di Cartier cfr. Pavanello, "From the 'Devil's Trill'."

${ }^{17}$ Su questa sonata cfr. Pavanello, "Il Trillo del diavolo."

${ }^{18}$ Charles Henri de Blainville trascrisse le sonate op. 1 Le Cène in forma di concerto grosso, cfr. Blainville, Concerti Grossi. Questa edizione è citata nel catalogo di Le Clerc databile tra il 1752 e il 1760, cfr. Devriès e Lesure, Dictionnaire des éditeurs, 1, Catalogues, n. 131.

${ }^{19}$ Tartini, Traité des agrémens.

20 "Lettera del defonto Signor Giuseppe Tartini alla Signora Maddalena Lombardini" e le traduzioni in inglese e francese.

${ }^{21}$ Mercure de France, dicembre 1747, 122; un mese prima, per la festa di tutti i Santi, un altro allievo di Tartini, Petit, si era esibito eseguendo una sonata a violino solo del maestro, cfr. Mercure de France, novembre 1747, 123.

${ }^{22}$ Mercure de France, febbraio 1748, 130.

${ }^{23}$ Mercure de France, novembre 1748, 168. 
e poi "M. Pagin a joué la Tempête di Mare del Signor Vivaldi", ${ }^{24}$

- 24 dicembre 1749, Pagin “joua seul”, il 25 dicembre suona "un Concerto"; 25

- marzo 1750, la domenica delle palme Pagin suona solo, mercoledì santo suona "avec applaudissement" e si esibisce anche il giorno di Pasqua (29 marzo). ${ }^{26}$

Pagin non era il solo a presentarsi al pubblico del Concert Spirituel suonando musiche di Tartini: Les Affiches de Paris narrano che il 24 dicembre 1741 "le Sr Petit exécuta un Concerto di Sr Tartini"; lo stesso periodico del 30 ottobre 1747 ci testimonia che il primo novembre, festa di tutti I Santi, "M. Petit exécutera una sonate à violon seul de M. Tartini". ${ }^{27}$ Oltre a Petit, ${ }^{28}$ lui stesso allievo di Tartini, anche Pierre Gaviniès si esibiva nel repertorio tartiniano: nel concerto del 2 febbraio 1751 "joua seul en maître une Sonate de M. Tartini”. Sembra che gli anni in cui a Parigi vennero stampate le raccolte di sonate tartiniane furono anche quelli in cui la musica del compositore istriano fu eseguita più frequentemente al Concert Spirituel. Tuttavia pare evidente che vi fossero orientamenti stilistici in contrasto con lo 'stile italiano' e che talvolta queste spinte prevalessero, poiché dopo le sue esibizioni durante le festività di Pasqua del 1750 non si ha più notizia della presenza di Pagin al Concert Spirituel e alcune celebri testimonianze attribuiscono la sua uscita di scena all'eccessiva italianità: si vedano a tal proposito le lettere di Grimm sull'Omphale ${ }^{29} \mathrm{e}$ la lettera di Rousseau - Lettre à M. Grimm, au sujet des remarques ajoutées à sa lettre sur Omphale $^{30}$-, tutte apparse nel 1752, che contribuirono a far deflagrare la polemica tra sostenitori della musica italiana e paladini della musica francese; si legga inoltre quanto

${ }^{24}$ Mercure de France, giugno 1749, 195.

${ }^{25}$ Mercure de France, gennaio 1750, 194-195.

${ }^{26}$ Mercure de France, aprile 1750, 184-186.

${ }^{27}$ La stessa notizia appare sul Mercure de France, novembre 1747, 123.

${ }^{28}$ Non si conosce il nome di questo violinista.

${ }^{29}$ Grimm, Lettre de M. Grimm sur Omphale; Id., Lettre de M. Grimm à l’Abbé Raynal: "J'ose enfin l'assurer que personne n'admire plus que moi le talent de l'élève du grand Tartini; mais je n'ai pas cru devoir rappeler au public un évènement qu'il pourrait trouver aujourd'hui beaucoup plus humiliant pour son goût, qu'il ne le fut alors pour le talent de M. Pagin." L'anonimo autore delle Remarques au sujet de la Lettre de M. Grimm sur Omphale aveva riferito della cattiva accoglienza ricevuta da Pagin al Concert Spirituel, 25-26: "J'aurois eu du plaisir à lire dans votre Lettre jusqu'à quel point la nation ingrate envers un talent si sublime, a osé l'humilier publiquement. C'est-là qu'il falloit prendre le ton de vos Philosophes, \& dire que l'amour de son talent lui a fait passer les Alpes, pour chercher un maître digne de lui. C'est la Musique de ce Maître qui inspire de nobles sensations. Original en tout, il n'a reçu de loix que celles de son génie. La sublimité de ses Ouvrages le fait estimer de tout homme capable de sentir la vérité. Il a su bannir le fard de la Musique pour servir la nature. Il est connu dans toute Europe, mais chèri de peu de gens, comme aussi peu de gens ont le bonheur de distinguer le bruit, d'avec le sentiment et l'expression. Tel est le maître que M. Pagin a fidèllement suivi dans ses études."

${ }^{30}$ [Rousseau], Lettre à M. Grimm; anche Rousseau, entrando nella controversia in difesa di Grimm sotto anonimato, fa riferimento a Pagin: "Le Commentateur s'étend sur l'éloge de Pagin et de son illustre maître, et nous y applaudissons vous et moi de très bon cœur. Il voudrait que vous eussiez dit jusqu'à quel point la nation ingrate envers un talent si sublime, a osé l'humilier publiquement. Il fallait dire, s'humilier publiquement. Midas n'humilia point Apollon, et un cygne peut être hué par des oies sans en être humilié." Tutti i testi cui si fa qui riferimento sono contenuti in $L a$ querelle des bouffons, curata da D. Launay. 
riportato da d'Aquin nel Siècle littéraire de Louis XV ou lettres sur les hommes célèbres ${ }^{31}$ e, qualche anno più tardi, da Burney in The Present State of Music in France and Italy. ${ }^{32}$ Tuttavia le motivazioni dell'allontanamento di Pagin dal Concert Spirituel non devono essere state solo queste, se si considera che dopo il 1750 Gaviniès si esibì da solo proprio con una sonata di Tartini e che negli stessi giorni in cui Pagin suonava per l'ultima volta al Concert Spirituel, qui il pubblico ebbe modo di ascoltare ed applaudire il violinista e compositore Felice Giardini. ${ }^{33}$ Forse la causa dell'isolamento di Pagin risiede nel fatto che il suo repertorio era limitato alla musica italiana, ${ }^{34}$ mentre Gaviniès, assai lodato proprio per questo da d'Aquin, ${ }^{35}$ si esibiva suonando sia musica francese che italiana; oppure, più probabilmente, l'astro nascente di Gaviniès aveva oscurato quello di Pagin, mentre musicisti e interpreti stranieri, solo di passaggio al Concert Spirituel, non furono toccati dalla concorrenza con gli interpreti locali. Una ragione potrebbe anche risiedere proprio nell'essere stato Pagin allievo di Tartini e nell'ammirazione che il compositore riscuoteva

${ }^{31}$ D'Aquin de Château-Lyon, Siècle littéraire de Louis XV, 136-137: "Parmi les Ecoliers du fameux Tartini, qu'on regarde toujours comme un prodige en Italie, \& qui quelquefois est critiqué en France, quoique le plus souvent admiré; parmi les écoliers de ce grand Maître Mr. Pagin tient, sans contredit, la premiere place. Il doit tout à Tartini; il s'est formé sous ses yeux; il ne connoît que lui; il ne joue que sa Musique, parce qu'elle est la seule qui lui paroisse touchante \& sublime. Mais ce jeune \& admirable Artiste, si surprenant dans son Art, si aimable dans la société, ne devroit-il pas se prêter un peu à tous les goûts? N'est-il pas fait pour embellir toutes les Musiques? D'ailleurs comme le dit le Poëte, quelquefois, Sur le ton des François il faut chanter en France."

${ }^{32}$ Burney, The Present State of Music, 42-44: "I heard M. Pagin on the violin, at the house of Madame Brillon, at Passy; she is one of the greatest lady-players on the harpsichord in Europe. This lady not only plays the most difficult pieces with great precision, taste, and feeling, but is an excellent sight's-woman: of which I was convinced by her manner of executing some of my own music, that I had the honour of presenting to her. She likewise composes; and was so obliging as to play several of her own sonatas, both on the harpsichord and piano forte, accompanied on the violin by M. Pagin. [...] M. Pagin was a pupil of Tartini, and is regarded here as his best scholar; he has a great deal of expression and facility of executing difficulties; but whether he did not exert himself, as the room was not large, or from whatever cause it proceeded, I know not, but his tone was not powerful. Music is now no longer his profession; he has a place under the Comte de Clermont, of about two hundred and fifty pounds sterling a year. He had the honour of being hissed at the Concert Spirituel for daring to play in the Italian style, and this was the reason of his quitting the profession."

${ }^{33}$ Mercure de France, aprile 1750, 184-186. Sul violinista torinese cfr. Hogwood e McVeigh, "Giardini."

${ }^{34}$ È quanto afferma Choron, Dictionnaire historique des musiciens, 2, 111: "Pagin joua plusieurs fois au Concert Spirituel avec le plus grand succès. Mais, comme il n'éxécutait que de la musique de Tartini, les musiciens français se liguèrent contre lui; et les applaudissements ironiques qu'il réçu un jour au Concert Spirituel le déterminèrent à n'y plus reparaître;" sull'argomento cfr. anche Desmet, "Tartini e la Francia." Tutta la vicenda di Pagin è rievocata in Charlton, Opera in the Age of Rousseau, 193-195; purtroppo Charlton dà a Pagin il nome errato di Jean-Pierre.

${ }^{35}$ D'Aquin de Château-Lyon, Siècle littéraire de Louis XV, 139: "Je vois un jeune Amphion qui va tout mettre d'accord. Mr. Gaviniés paroît, il n'est point éleve de Tartini, mais formé par la nature \& l'art, pour aspirer à la premiere place, il prend son violon, prélude: Quels sons vous entendez! quel coup d'archet! que de force! que de grace! c'est Baptiste lui-même. Je suis saisi, enchanté, il parle à mon coeur, tout brûle en ses mains. L'Italien, le François, il l'exécute avec le même nerf \& la même precision." 
nell'ambiente dei philosophes; ${ }^{36}$ le polemiche tra sostenitori della musica italiana e della musica francese potrebbero aver nuociuto alla fama del secondo e alla carriera del primo.

L'op. 4 di Tartini, apparsa nel 1747, è dedicata a Pagin ${ }^{37}$ e l'anno successivo l'allievo pubblicò la sua prima raccolta, Sonates à violon seul et basse continue. ${ }^{38}$ Anche l'op. 5 è dedicata al violinista francese - la dedica, a firma dello stampatore Hue, è la medesima che compare nell'edizione precedente - mentre l'op. 7, seconda edizione, appare personalmente curata da Pagin, come si rileva dall'Avvertissement; in questa stampa il violinista francese appronta una propria versione ornamentale del secondo movimento della terza sonata (G9, vedi Fig. 1):

Ce ne sont pas seulement des Sonates du Celebre Tartini que je presente au public en seconde Edition mais je les presente revües avec tous les soins et la sagacité de Monsieur Pagin. La frequentation qu'il à eû avec ce grand harmoniste dans les années qu'il a passé en Italie, la facilité avec laquelle il a saisi et son gout et son toucher authorisent l'espoir que j'ai d'avoir rempli les attentes des amateurs des ouvrages de cette merveille de l'Italie. Si la modestie de Monsieur Pagin ne m'imposoit silence; j'oserois avancer à haute voix que j'ai la certitude du contentement du public en voyant a la tête de cet Euvre le nom d'un homme qui par ses talens et sa profonde connoissance de touttes les parties necessaires à un harmoniste du premiere ordre s'est etabli une reputation d'autant plus inoüie, que le siecle où nous vivons est plus delicat et cette reputation est d'autant plus juste qu'il ne voit tout au plus que des Rivaux dans ce même siecle où il s'etoit elevé plusieurs genies qui ont passé pour des chefs-d'œuvre dans son art. Les curieux trouveront dans cette seconde Edition deux Sonates qui n'etoient point dans la premiere.

Se appare evidente un coinvolgimento di Pagin nella serie di edizioni dall'op. 4 all'op. 7, più difficile è definirne la portata: il suo nome serviva ad accrescere l'appetibilità delle stampe tartiniane grazie alla notorietà che costui aveva raggiunto con le esibizioni al Concert Spirituel, oppure Pagin ebbe il ruolo di fornire all'editore le sonate di Tartini da pubblicare?

Che gli allievi di Tartini fossero in possesso di numerose composizioni manoscritte del maestro è fuor di dubbio, tuttavia non si può affermare con certezza che Pagin avesse in questo caso consegnato agli editori le sonate; il repertorio contenuto nelle stampe

${ }^{36}$ Sui rapporti tra il pensiero di Rousseau e Tartini cfr. Felici, “"Non suona, canta su'l violino';" cfr. anche Boccadoro, "Tartini, Rousseau et les Lumières."

${ }^{37}$ Tartini, Sonates a Violon Seul avec la Basse Continue [... . Euvre IV. e: "A Monsieur Pagin Virtuos. ${ }^{m o}$ Di Violino. Monsieur, Je prends la liberté de Vous presenter ce livre de Sonates de votre très Excellent Maître Monsieur Giuseppe Tartini, pourois-je l'offrir a quelqu'un qui en connoisse mieux les Beautés, et qui puisse le faire valloir autant que vous. Je saisis avec empressement cette occasion pour rendre à vos talents éminents la justice qui leur est düe, et pour vous assurer des parfaits sentiments avec les quels j'ay l'honneur d'être, Monsieur, Votre tres humble, et tres obeissant serviteur, L. Hue."

${ }^{38}$ Pagin, Sonates a violon seul et basse-continue. La stampa fu annunciata in Les Affiches de Paris, Avis divers, 6 giugno 1748, e nel Mercure de France, giugno 1748, 149; cfr. Devriès-Lesure, L'édition musicale dans la presse parisienne, 390, dove tuttavia anche Devriès dà a Pagin il nome errato di Pierre. 
francesi potrebbe essere giunto nelle mani di Le Clerc e degli altri stampatori anche per altre vie, infatti più d'uno furono gli allievi francesi di Tartini. ${ }^{39}$

Grazie al confronto con gli annunci apparsi nelle riviste coeve (Mercure de France, Affiches de Paris, Annonces, affiches, et avis divers) e nei cataloghi degli editori, spesso acclusi nelle edizioni stesse, possiamo ricostruire con buona approssimazione il lasso di tempo in cui apparvero le edizioni francesi di sonate tartiniante. Sorprendentemente, dall'op. 3 (ristampa dell'edizione romana op. 2) all'op. 9, come si può vedere dalla Tavola 1, le stampe di sonate tartiniane apparvero tutte in un arco di tempo di soli tre anni, dal 1747 al 1750, che coincide, forse non del tutto casualmente, con il periodo in cui Pagin si esibì regolarmente al Concert Spirituel. Anche se certamente le sonate in questione andarono incontro a ristampe negli anni successivi, tuttavia dall'Italia non dovettero provenire altre composizioni, con le significative eccezioni de L'Arte dell'arco - nella versione ampliata con 38 variazioni - e della sonata nota come Il trillo del diavolo, che avrebbero costituito le basi della fortuna ottocentesca di Tartini; solo alla fine del secolo, nel periodo napoleonico e in conseguenza dell'occupazione francese dell'Italia, sarebbero giunti in Francia i numerosi manoscritti tartiniani redatti principalmente da Giulio Meneghini, ${ }^{40}$ oggi alla Biblioteca Nazionale di Parigi (Fondo del Conservatorio).

A un esame delle sonate in questione e delle loro concordanze manoscritte risultano alcuni elementi interessanti. Le principali fonti manoscritte contenenti il corpus delle sonate tartiniane sono: l'autografo conservato presso l'archivio della Cappella Antoniana di Padova, I-Pca 1888 fasc. 1 (contenente 31 sonate complete più alcuni movimenti isolati e abbozzi); il manoscritto I-Pca 1905 (contenente 61 sonate di cui 3 autografe); il manoscritto conservato presso la Biblioteca Nazionale di Parigi, F-Pc 9796 (contenente 51 sonate, di cui 9 autografe, e L'Arte dell'arco).

Nell'op. 4 tre delle sei sonate (E3, B10, A3) compaiono in numerose copie manoscritte e due (E3, A3) sono incluse nell'elenco di sonate e concerti tartiniani redatto da Giulio Meneghini, ${ }^{41}$ per di più di esse si conserva l'autografo (rispettivamente F-Pc 9796/6 e

${ }^{39}$ Oltre al già citato Petit, studiarono con Tartini Pierre Lahoussaye, Joseph Touchemoulin e de Tremais; cfr. Capri, Giuseppe Tartini, 384-404.

${ }^{40}$ Giulio Meneghini fu violinista e copista della Cappella Antoniana di Padova a partire dal 1756 e poi successore di Tartini. I manoscritti che costituiscono il fondo destinato al Conservatorio di Parigi e ora alla Bibliothèque Nationale sono stati raccolti, copiati o integrati da Meneghini alla fine del Settecento, durante l'occupazione francese di Padova, per essere consegnati "parte al Cittadin Pagnini, e Parte al Comis:o Berthollet," come si legge nel catalogo apposto al ms. F-Pc 9796. Claude-Louis Berthollet, chimico originario della Savoia, dal 1796 fece parte della commissione che ebbe l'incarico di requisire le opere d'arte da inviare in Francia (dove giunsero in trionfo il 27 luglio 1798).

${ }^{41}$ Meneghini ha redatto i seguenti manoscritti di sonate tartiniane: A-Wgm ms. IX 33956 (copia pressoché integrale del manoscritto autografo I-Pca 1888/1), F-Pc ms. 951, 14 delle 61 sonate del manoscritto I-Pca 1905 (le pagine contenenti queste sonate appartenevano in origine al manoscritto A-Wgm, ms. IX 33956, pp. 33-40, ove sono state sostituite da copie di mano diversa da quella di Meneghini). Meneghini ha inoltre redatto il catalogo contenuto alle pp. 407-410 del manoscritto F-Pc 9796, in cui le sonate sono divise cronologicamente in tre gruppi: nn. 1-10 ("Vecchie, ho sia composte nei primi suoi anni"), 11-37 ("Meno vecchie"), 38-43 ("Segue ultime recenti composte negl'ultimi anni della sua vitta cioè dell'anno 1765, e 1766"). Oltre agli incipit di queste sonate, Meneghini cita "Altre Sonate a Violino e Basso, cioè. Opera Prima Sonate 
9796/3) e la sonata A3 appare anche nell'op. 1 Witvogel. Le sonate G20, A3 e D15 sono conservate tra l'altro nella collezione della Music Library dell'Università di Berkeley ${ }^{42}$ (US-BEm item 750, 772 e 713) espressamente attribuite a Tartini. L'ultima composizione della stampa non appare invece in alcuna fonte manoscritta, ma in una edizione di composizioni di Mauro d'Alay apparsa a Londra nel 1728 (quasi vent'anni prima): ${ }^{43}$ si potrebbe ipotizzare che l'editore, disponendo di sole cinque sonate tartiniane, abbia voluto completare la raccolta inserendovi una composizione di un altro autore italiano; in questo caso sembra difficile che Pagin abbia potuto prendere parte alla predisposizione dei brani. È interessante notare come nei cataloghi dello stampatore Hue, subito prima delle opere tartiniane pubblicate dallo stesso, compaia una raccolta di sonate per violino op. 1 proprio di Mauro d'Alay (nella grafia Dallai), forse una ristampa delle sonate già apparse a Londra insieme alle cantate.

Riguardo l'op. 5 abbiamo concordanze per le prime quattro composizioni, mentre per le ultime due (F10, B12) non vi è alcuna fonte manoscritta. Nell'op. 6 tutte le sonate tranne la seconda (A19) hanno numerose concordanze e, a differenza delle edizioni precedenti, sono state copiate anche nel manoscritto I-Pca 1905. Delle cinque sonate dotate di concordanze, tre sono trasmesse anche nel manoscritto F-Pc 9796 e appaiono incluse nel catalogo delle opere di Tartini redatto da Giulio Meneghini.

La stessa relazione con i manoscritti F-Pc 9796 e I-Pca 1905 si ritrova nell'op. 7, ove le sonate presentano tutte numerose concordanze, tranne la seconda (B7), che corrisponde alla sonata $\mathrm{n}$. 1 dell'op. 1 Witvogel, seppur con il primo movimento diverso: questa sonata non compare in alcuna fonte manoscritta. Nella seconda edizione dell'op. 7 due sonate sono state sostituite con altre (rispettivamente E4 con a7 e F7 con D10), anche queste ultime concordanti con i manoscritti I-Pca 1905 e F-Pc 9796.

L'op. 8 corrisponde ad un'edizione di sonate a tre, ${ }^{44}$ mentre l'op. 9 ci offre una situazione in qualche modo simile all'op. 4 e all'op. 5, poiché solo tre sonate (Es1, G6, F6) presentano numerose concordanze, mentre la G22 è una versione diversa di quella contenuta nell'op. 4 e le sonate A20 e D16 sono prive di concordanze.

Nella Tavola 2 ho annotato le concordanze, qualora ve ne siano, con i manoscritti I-Pca 1905 e F-Pc 9796, e l'inclusione o meno delle sonate edite in Francia nel catalogo di Meneghini: come si può vedere dal confronto con il catalogo (la cui numerazione segue un ordine cronologico), nelle edizioni francesi non si riscontra alcuna relazione tra la cronologia delle sonate e l'ordine della loro pubblicazione; anche nel manoscritto I-Pca1905 non vi è alcuna corrispondenza con la successione delle sonate nel Catalogo, forse a causa della sua organizzazione in fascicoli assemblati successivamente alla loro redazione. Degno di nota è il fatto che nessuna delle sonate pubblicate concordi con il manoscritto autografo I-Pca 1888 fasc. 1, che costituisce la fonte più importante per le

N:o 12 Stampate /in Abstardan [sic]. Opera Seconda Sonate N:o 12 Stampate in Roma. Opera Terza manoscritta in libro con Sonate N:o 28, e con molte agiunte in fine, adatabile alle Sonate stesse. Viariazioni [sic] sopra la Gavotta del Corelli" (cfr. Canale Degrassi, "I concerti solistici di Giuseppe Tartini," 1, 6).

${ }^{42} \mathrm{Su}$ questa collezione cfr. Duckles e Elmer, Thematic Catalog.

${ }^{43}$ D'Alay, Cantate a voce sola.

${ }^{44}$ Sul repertorio tartiniano di sonate a tre cfr. Brainard, "Le Sonate a tre." 
sonate solistiche di Tartini; forse ciò potrebbe suffragare l'ipotesi di Brainard che la sua compilazione sia cominciata non prima del 1745 per estendersi poi nell'arco di molti anni. ${ }^{45}$

Una comparazione dettagliata tra le fonti manoscritte e le edizioni francesi di sonate tartiniane esula da questo articolo, tuttavia si può osservare come in generale le sonate contenute nelle edizioni dall'op. 4 all'op. 9 presentino costantemente la notazione della numerica del basso continuo, benché non sempre coincidente con quella di altre fonti, e numerose indicazioni di fraseggio. Per quanto riguarda gli elementi ornamentali, prevalgono il simbolo per il trillo, l'appoggiatura e l'inserimento del ritmo puntato; ad esempio la prima sonata dell'op. 6, G8, presenta nell'Adagio introduttivo un massiccio uso del ritmo puntato, laddove la copia manoscritta in US-BEm item 742 ne è del tutto priva; l'inegalité è però presente nella versione trasmessa nel manoscritto I-Pca 1896/15, con inserti autografi - in cui sono sovrapposte diverse soluzioni ornamentali per lo stesso movimento - a conferma che tale prassi doveva essere consueta nell'ambito della Scuola delle Nazioni. Troviamo anche il ritmo puntato costituito da una nota breve seguita da una nota lunga, il cosiddetto ritmo lombardo, in particolare nell'Allegro assai conclusivo della Sonata n. 5 dell'op. 4, D15. Come abbiamo visto, alcune sonate presentano movimenti che non compaiono in altre fonti, ad esempio la Sonata G6, op. 9 n. 2, ha il primo movimento diverso da quello attestato nell'autografo F-Pc 9796/4, la Sonata G21, op. 5 n. 4 , ha il terzo movimento, Allegro assai, diverso dalle altre due fonti note (US-BEm item 751 e S-Skma C 1-R), mentre la Sonata G22, op. 9 n. 3, presenta un primo movimento non attestato in altre fonti e ha come ulteriori tre movimenti quelli che compongono la sonata G20, già apparsa a stampa nell'op. 4.

Le numerose concordanze delle stampe francesi op. 6 e op. 7 confermano l'attendibilità di queste edizioni, malgrado la mancanza di testimonianze relative a contatti tra gli stampatori e Tartini. In generale, l'assenza di concordanze manoscritte per alcune delle sonate apparse nelle stampe dall'op. 4 all'op. 9 non può essere considerata automaticamente indizio della loro inautenticità, come invece sembrerebbe presupporre Brainard; 46 proprio la presenza in Francia di allievi e ammiratori della musica di Tartini può essere considerata un deterrente alla falsificazione da parte di editori privi di scrupoli, che tuttavia non si può escludere del tutto.

La frequenza delle esecuzioni di sonate e concerti tartiniani al Concert Spirituel, l'attività di violinisti e compositori suoi allievi, il numero di stampe contenenti opere di Tartini che videro la luce nel giro di pochi anni, la frequente citazione del suo nome negli scritti e pamphlets dell'epoca ci permettono di tracciare l'apice della fortuna di Tartini in Francia tra gli anni Quaranta e gli anni Sessanta del Settecento. Sappiamo che negli anni Ottanta le fortune di Tartini, almeno in ambito concertistico, erano assai scemate, se si considera il giudizio espresso nei confronti di Maddalena Lombardini Sirmen, la cui esibizione al Concert Spirituel venne criticata proprio in virtù dell'essere troppo legata al repertorio e alla prassi esecutiva del suo maestro. ${ }^{47}$ Tuttavia, quando alla fine del secolo

${ }^{45}$ Cfr. Brainard, "Introduzione."

${ }^{46}$ Cfr. Brainard, "Le Sonate a tre," 108: "Nessun diretto contatto è mai stato accertato fra Tartini e gli editori Maupetit-Boivin-LeClerc, e il lungo elenco delle loro pubblicazioni con il nome di Tartini contiene parecchie opere che sono quasi certamente spurie."

${ }^{47}$ Mercure de France, 7 maggio 1785, 76-77: "M.me Siremen, qui s'étoit fait entendre ici sur le 
si vorranno porre le basi del Conservatoire de Musique parigino, è proprio alla Scuola delle Nazioni e al suo repertorio che si guarderà come modello musicale e pedagogico.

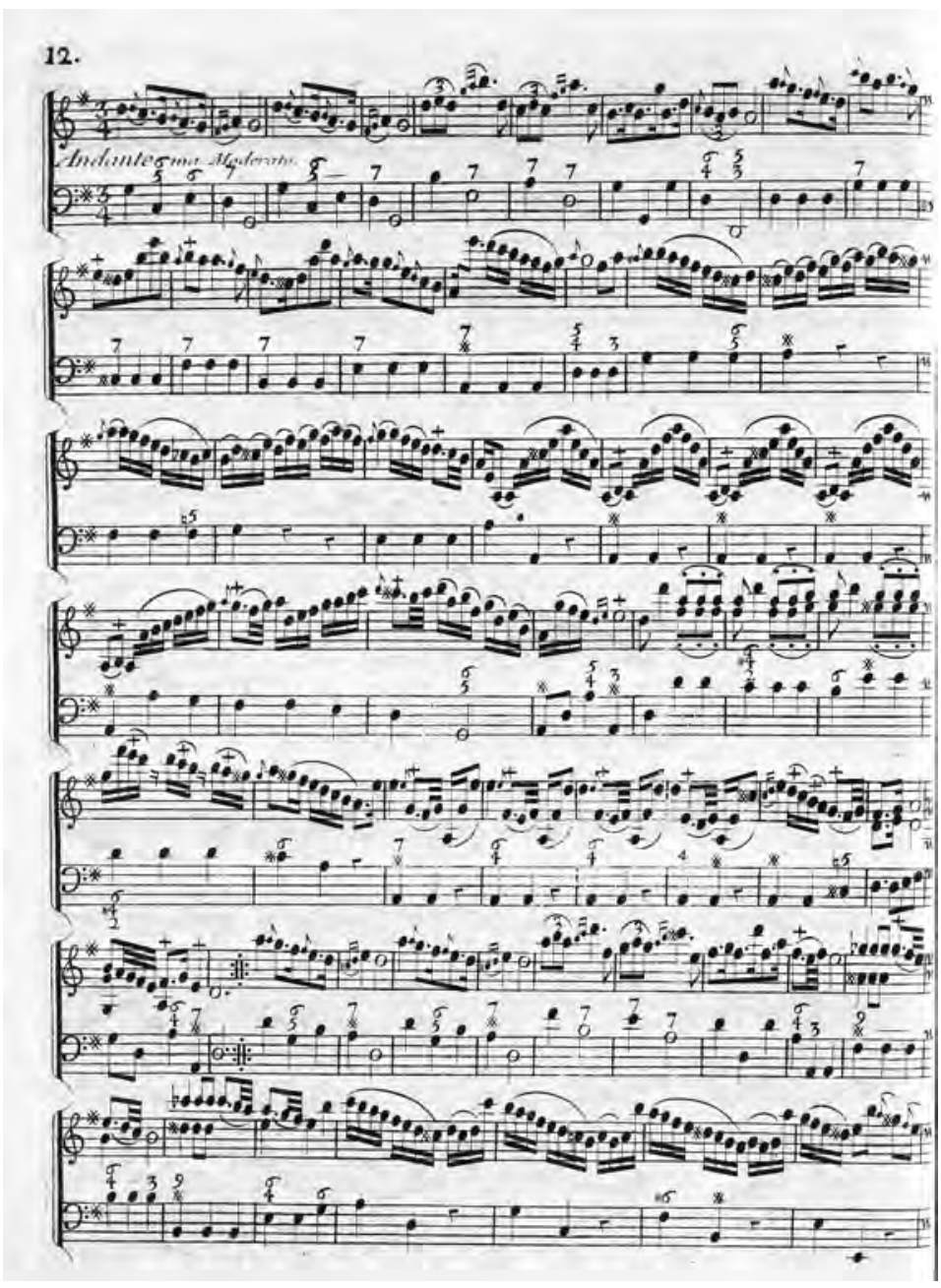

Figura 1

Giuseppe Tartini, Sonate a violino solo col basso, op. 7, seconda edizione riveduta, Sonata III, mov. 2, Andante ma moderato, p. 12 (Museo internazionale e biblioteca della musica di Bologna; per gentile concessione)

violon, il y a quatorze ans, a reparu de nouveau; mais on ne peut dissimuler que la sensation qu'elle a produite n'ait été moins favorable. Madame Siremen a conservé les principes de l'eccellente École de Tartini, peut-être trop oubliés aujourd'hui, une charmante qualité de son, de beaux doigts, un jeu plein d'intérêt $\&$ de grâce [...] mais son style, le même qu'elle avait il y a quatorze ans, est extrêmement vieilli; depuis qu'on a substitué des notes à des sons, des tours de force à des traits de chants; on ne veut plus qu'étonner [...] enfin [...] nous croyons devoir lui conseiller de jouer des Concertos d'un style plus moderne, \& nous ne doutons pas qu'alors elle ne ramène autant de suffrages qu'elle en a obtenus autrefois." 


\section{Tavola 1. Edizioni di opere tartiniane per violino e basso}

Op. 1

Edizione: Amsterdam: Witvogel, [1730-1731]. RISM: T 240

Annunci:

Ristampe:

Contenuto: $\quad$ B7, a9, h5, g9, A3, B8

Op.1

Edizione: Amsterdam: Michel Charles Le Cène, 1734 [frontespizio datato]. RISM: T 241

Annunci: $\quad$ Amsterdamsche Courant, 25 giugno, 2, 11 luglio 1733

Amsterdamsche Courant, 9, 16 maggio 1734

Ristampe: ${ }^{48}$ Paris: Le Clerc, [1744]. ${ }^{49}$ RISM: T 244-245

Paris: Imbault, [ca. 1749]. RISM: T 246

Paris: Conservatoire n. 137. RISM: T 247

Paris: Janet \& Cotelle, n. 137. RISM: T 248

Contenuto: A14, F9, C11, G17, e6, D12, D6, c2, A15, g10, E5, F4, A16 (Pastorale)

Op. 2

VI Sonate a Violino e Violoncello o Cimbalo Opera Seconda di Giuseppe Tartini di Padoa

Edizione: Amsterdam: Michel Charles Le Cène, [1743]. RISM: T 251

Annunci: $\quad$ Gazette d'Amsterdam, 18 ottobre 1743

Ristampe: Paris: Le Clerc, [1744]. RISM: T 253-254

Paris: Janet \& Cotelle. RISM: T 255

Contenuto: $\quad$ g4, A5, d4, e7, F5, E6

Op. 2

Sonate a Violino e Basso dedicate al S. ${ }^{r}$ Guglielmo Fegeri di Giuseppe Tartini Opera Seconda

Edizione: Roma: Cleton, 1745 [dedica datata «Padova li 30 Giugno 1745»]. RISM: T 257

Annunci:

Ristampe: Paris: Le Clerc, [1747], op. 3. ${ }^{50}$ RISM: T 260

Contenuto: D13, G18, A17, h6, a10, C12, g11, D14, B9, F8, e8, G19

\section{L'arte dell'arco}

Nouvelle étude pour le violon ou Manière de varier et orner un pièce dans le gout du Cantabile Italien. par M. ${ }^{r}$ Pétronio Pinelli virtuoso Romano. Augmenté d'une Gavotte de Corelli, travaillez et doublez Par M. ${ }^{r}$ Giuseppe Tartini

Edizione: Paris: Hue, [ante o nel 1747]. ${ }^{51}$ RISM: T 277

${ }^{48}$ Sono qui citate solo le ristampe francesi.

${ }^{49}$ Edizione citata nel catalogo dell'editore Charles-Nicolas Le Clerc pubblicato nel 1744; cfr. Devriès e Lesure, Dictionnaire des éditeurs de musique français, 1, Catalogues, n. 127.

${ }^{50}$ Edizione citata nel catalogo di Le Clerc pubblicato attorno al 1747; cfr. ivi, n. 128.

${ }^{51}$ La Nouvelle étude pour le violon, et une Gavotte de Corelli, l'op. 4 e l'op. 5 sono tutte citate nel catalogo di Hue pubblicato attorno al 1747; cfr. ivi, n. 102. 
Annunci:

Ristampe:

Contenuto: F11 [17 variazioni]

Op. 4

Edizione: $\quad$ Paris: Hue, [ca. 1747]. RISM: T 264

Annunci:

Ristampe:

Contenuto: E3, G20, B10, A3, D15, c5

Op. 5

Edizione:

Sonates A violon seul avec la Basse Continue. Composées par M. ${ }^{r}$ Giuseppe Tartini di Padoa dédiées A Monsieur Pagin oeuvre Ve

Annunci:

Ristampe:

Contenuto: a11, B11, A18, G21, F10, B12

Op. 6

Edizione:

Sei Sonate a violino e violoncello O Cimbalo di Giuseppe Tartini di Padoa opera VI Annunci:

Ristampe:

Contenuto: G8, A19, D8, A6, B5, G10

Op. 7

Edizione: Paris: Maupetit, ${ }^{53}$ [1748]. RISM: T 268

Annunci: $\quad$ Les Affiches de Paris, 1 luglio 1748

Ristampe:

Sonate A Violino Solo Col Basso Da Signore Giuseppe Tartini. di Padoua opera VII

Contenuto:

D11, B7, G9, E4, g3, F7

\section{Op. 7 Seconda edizione riveduta}

Sonate a violino solo Col Basso Del Signor Giuseppe Tartini di Padoua Opera VII Seconde Edition Gravée par M. ${ }^{\text {elle }}$ Bertin

Edizione: $\quad$ Paris: Maupetit, [1748]. RISM: T 269

Annunci:

Ristampe:

Contenuto: D11, B7, G9, a7, g3, D10

52 L'op. 6 è citata nel catalogo di Le Clerc del 1748; anche in questo caso la datazione del catalogo al 1748-1749 proposta da Devriès e Lesure è da circoscrivere al 1748, poiché l'op. 6 non può essere posteriore all'op. 7 apparsa nel 1748; cfr. Devriès e Lesure, Dictionnaire des éditeurs de musique français, 1, Catalogues, 14.

${ }^{53}$ Questo editore è assente dal Dictionnaire di Devriès e Lesure. 
op. 8

Sei Sonate a tre, due violini col basso del signor Giuseppe Tartini di Padoa gravé par M.lle Bertin

Edizione: $\quad$ Paris: Maupetit, [1749]. RISM: T 271

Annunci: $\quad$ Les Affiches de Paris, 2 gennaio 1749

Ristampe:

Contenuto: A5+A9, D7, G4, D10, A10, D11

op. 9

Sei Sonate A Violino e Violoncello o Cimbalo. di Giuseppe Tartini di Padoa. Opera IX.

Edizione: $\quad$ Paris: Le Clerc, [1749-1750]. ${ }^{54}$ RISM: T 273

Annunci:

Ristampe:

Contenuto: Es1, G6, G22, A20, F6, D16

\section{L'arte dell'arco}

L'arte del arco ou l'art de l'archet

Edizione: $\quad$ Paris: Le Clerc, [1757]. RISM: T 278

Annunci: $\quad$ Annonces, affiches, et avis divers, 28 novembre 1757 e gennaio 1758

Mercure de France, gennaio 1758

Ristampe: $\quad$ Paris: Le Duc, n. 99. RISM: T 279

Contenuto: F11 [38 variazioni]

\section{Jean Baptiste Cartier, L'Art du Violon}

L'art du violon, ou Collection choisie dans les Sonates des Ecoles Italienne, Françoise et Allemande, précédée d'un abrégé de principes pour cet instrument; Dédié au Conservatoire de Musique

Edizione: Paris: Decombe, [1798]. RISM BVI p. 209

Annunci:

Ristampe: $\quad$ Paris: Decombe, 1801 e ca. 1803 (edizione rivista e corretta; F 11 con 50 variazioni)

Contenuto: g4, A14, F11 [38 variazioni; 50 variazioni], g5, F5 [mov. 1 con 17 variazioni]

${ }^{54}$ La stampa si trova compresa nel catalogo di Le Clerc redatto tra il luglio 1749 e il giugno 1750 , cfr. Devriès e Lesure, Dictionnaire des éditeurs de musique français, 1, Catalogues, n. 130. Brainard aveva invece datato l'op. 9 attorno agli anni Sessanta (cfr. Brainard, Le sonate per violino, XXXVIII), poiché nel catalogo contenuto in questa stampa compare L'arte del arco, databile al 1757; tuttavia l'esemplare consultato da Brainard, contenente il catalogo, potrebbe essere una ristampa dell'op. 9 posteriore al 1757. 
Tavola 2. Principali concordanze manoscritte delle edizioni di sonate tartiniane

\begin{tabular}{|c|c|c|}
\hline $\begin{array}{l}\text { Op. 1 Witvogel, } \\
\text { [1730-1731] }\end{array}$ & $\begin{array}{l}\mathrm{B} 7, \text { a9, }{ }^{1} \text { h5, g9, } \\
\underline{\mathrm{A} 3}{ }^{2} \underline{\mathrm{B} 8}\end{array}$ & $\begin{array}{l}\text { A3, F-Pc Catalogo n. 2, autografo F-Pc 9796/3, I-Pca } \\
1890 \\
\text { B8, F-Pc Catalogo n. 27, I-Pca 1905/47, F-Pc 9796/17 }\end{array}$ \\
\hline $\begin{array}{l}\text { Op. } 1 \text { Le Cène, } \\
1734\end{array}$ & $\begin{array}{l}\text { A14, F9, C11, G17, } \\
\text { e6, D12, D6, c2, } \\
\text { A15, g10, E5, F4, } \\
\text { A16 (Pastorale) }\end{array}$ & $\begin{array}{l}\text { D6, autografo I-Pca 1905/2 } \\
\text { F4, autografo I-Pca 1905/1, F-Pc 9796/11 }\end{array}$ \\
\hline $\begin{array}{l}\text { Op. } 2 \text { Le Cène, } \\
1743\end{array}$ & $\frac{\mathrm{g} 4}{\mathrm{~F} 5}, \underline{\mathrm{A} 5}, \underline{\mathrm{E} 6}, \underline{\mathrm{d} 4}, \underline{\mathrm{e} 7}$ & $\begin{array}{l}\text { g4, F-Pc Catalogo 32, I-Pca 1905/33, F-Pc 9796/43 } \\
\text { A5, F-Pc Catalogo 11, I-Pca 1905/21, F-Pc 9796/18 e } 40 \\
\text { d4, F-Pc Catalogo 21, I-Pca 1905/22 (2 copie), F-Pc } \\
\text { 9796/48 } \\
\text { e7, F-Pc Catalogo 24, I-Pca 1905/57, F-Pc 9796/35 } \\
\text { F5, F-Pc Catalogo 9, autografo F-Pc 9796/5 } \\
\text { E6, F-Pc Catalogo 14, I-Pca 1905/13, F-Pc 9796/45 }\end{array}$ \\
\hline $\begin{array}{l}\text { Op. } 2 \text { Cleton, } \\
1745\end{array}$ & $\begin{array}{l}\text { D13, G18, A17, } \\
\text { h6, a10, C12, g11, } \\
\text { D14, B9, F8, e8, } \\
\text { G19 }\end{array}$ & $\begin{array}{l}\text { G18, F-Pc } 9796 / 54 \text { e F-Pc } 951 \\
\text { C12, F-Pc 9796/55 e F-Pc } 951 \\
\text { B9, F-Pc 9796/51 } \\
\text { F8, I-Pca } 1905 / 38, \text { F-Pc } 9796 / 52\end{array}$ \\
\hline $\begin{array}{l}\text { L'arte dell'arco, } \\
\text { Hue [ca. 1747] }\end{array}$ & F11 [17 var.] & \\
\hline $\begin{array}{l}\text { Op. } 4 \text { Hue, } \\
{[1747]}\end{array}$ & $\begin{array}{l}\mathrm{E} 3, \mathrm{G} 20, \mathrm{~B} 10, A 3,{ }^{3} \\
\mathrm{D} 15, \mathbf{c} 5\end{array}$ & $\begin{array}{l}\text { E3, F-Pc Catalogo 8, autografo F-Pc } 9796 / 6 \\
\text { B10, F-Pc 9796/13 }\end{array}$ \\
\hline $\begin{array}{l}\text { Op. } 5 \text { Hue, } \\
{[1747]}\end{array}$ & $\begin{array}{l}\text { a11, B11, A18, } \\
\text { G21, F10, B12 }\end{array}$ & G21, due copie ms. con mov. 3 diverso \\
\hline $\begin{array}{l}\text { Op. 6, Le Clerc, } \\
{[1748]}\end{array}$ & $\begin{array}{l}\mathrm{G} 8, \mathrm{A19}, \underline{\mathrm{D} 8}, \underline{\mathrm{A} 6}, \\
\underline{\mathrm{B} 5}, \mathrm{G} 10\end{array}$ & $\begin{array}{l}\text { G8, I-Pca 1905/12 e I-Pca 1896/16 } \\
\text { D8, F-Pc Catalogo 13, I-Pca 1905/34, F-Pc 9796/29 } \\
\text { A6, F-Pc Catalogo 36, I-Pca 1905/26, F-Pc 9796/16 } \\
\text { B5, F-Pc Catalogo 25, I-Pca 1905/27, F-Pc 9796/36 } \\
\text { G10, I-Pca 1905/41 }\end{array}$ \\
\hline $\begin{array}{l}\text { Op. 7, Maupetit, } \\
{[1748]}\end{array}$ & $\begin{array}{l}\underline{\mathrm{D} 11}, B 7, \underline{\mathrm{G} 9}, \underline{\mathrm{E} 4}, \\
\mathrm{~g} 3, \underline{\mathrm{F} 7}\end{array}$ & $\begin{array}{l}\text { D11, F-Pc Catalogo 29, F-Pc 9796/39 e 9796/50 } \\
\text { G9, F-Pc Catalogo 18, I-Pca 1905/18 ( } 2 \text { copie) e 1896/8, } \\
\text { F-Pc 9796/47 } \\
\text { E4, F-Pc Catalogo 10, I-Pca 1905/32, autografo F-Pc } \\
\text { 9796/9 e 9796/12, I-Pca 1896/16 (autografo del mov. } 1 \\
\text { in } 3 \text { versioni ornate) } \\
\text { g3, I-Pca 1905/23, F-Pc 9796/49 } \\
\text { F7, F-Pc Catalogo 23, I-Pca 1905/24 (2 copie), F-Pc } \\
\text { 9796/33 }\end{array}$ \\
\hline $\begin{array}{l}\text { Op. 7, Seconda } \\
\text { edizione rivedu- } \\
\text { ta, Maupetit, } \\
\text { [1748] }\end{array}$ & $\begin{array}{l}\text { D11, B7, G9, } \underline{\mathrm{a} 7}, \\
g 3, \underline{\mathrm{D} 10}\end{array}$ & $\begin{array}{l}\text { a7, F-Pc Catalogo 30, I-Pca 1905/56, F-Pc 9796/44 } \\
\text { D10, F-Pc Catalogo 37, I-Pca 1905/20 (3 copie), F-Pc } \\
\text { 9796/37 }\end{array}$ \\
\hline $\begin{array}{l}\text { Op. 9, Le Clerc, } \\
{[1749-1750]}\end{array}$ & $\begin{array}{l}\text { Es1, G6, G22 } \\
\text { (mov. 2, 3, } 4= \\
\text { G20), A20, } \underline{\text { F6 }} \\
\text { D16 }\end{array}$ & $\begin{array}{l}\text { Es1, F-Pc Catalogo 34, I-Pca 1905/37, F-Pc 9796/42 } \\
\text { G6, F-Pc Catalogo 4, autografo F-Pc 9796/4 (mov. } 1 \\
\text { diverso) } \\
\text { F6, F-Pc Catalogo 1, I-Pca 1905/48, autografo F-Pc } \\
\text { 9796/1 }\end{array}$ \\
\hline
\end{tabular}




\begin{tabular}{|l|l|l|}
\hline $\begin{array}{l}\text { L'arte dell'arco, } \\
\text { Le Clerc, [1757] }\end{array}$ & F11 [38 var.] & \\
\hline $\begin{array}{l}\text { Cartier, L'art } \\
\text { du violon, De- } \\
\text { combe, [1798] }\end{array}$ & $\begin{array}{l}\text { g4, A14, F11, g5, } \\
\text { F5 (mov. 1 con 17 } \\
\text { variazioni) }\end{array}$ & g5, I-Pca 1905/35, F-Pc 9796/22 \\
\hline
\end{tabular}

1 Tutte le sonate la cui sigla è in grassetto sono prive di concordanze.

2 Ho sottolineato tutte le sonate presenti nel catalogo di Meneghini.

3 Ho posto in corsivo le sigle delle composizioni già apparse in edizioni precedenti.

\section{Bibliografia}

A Letter from the Late Signor Tartini to Signora Maddalena Lombardini (now Signora Sirmen), Published as an Important Lesson to Performers on the Violin. A cura di Charles Burney. London: Bremner, 1771.

Blainville, Charles Henri. Concerti Grossi Con due Violini, Viola e Violoncello di Concertino Obligati, e due altri Violini e Basso di Concerto Grosso da Carlo Blainville, Composti della Prima e Seconda parte dell'opera Prima di Giuseppe Tartini. Paris: Le Clerc, [s. a.].

Boccadoro, Brenno. "Tartini, Rousseau et les Lumières.” In Jean-Jacques Rousseau, Euvres complètes, 5, a cura di Marcel Raymond et al., 1694-1711. Paris: Gallimard, Bibliothèque de la Pléiade, 1995.

Brainard, Paul. “Introduzione.” In Giuseppe Tartini, La raccolta di sonate autografe per violino, facsimile, 7-27. Padova: Edizioni dell'Accademia Tartiniana, 1976.

___ L L sonate per violino di Giuseppe Tartini: catalogo tematico. Padova: Accademia Tartiniana; Milano: Carisch, 1975.

____ "Le Sonate a tre di Giuseppe Tartini: un sunto bibliografico." Rivista italiana di Musicologia 4 (1969): 102-126.

Burney, Charles. The present state of Music in France and Italy. London: Becket and Co., 1771.

Canale Degrassi, Margherita. "I concerti solistici di Giuseppe Tartini. Testimoni, tradizione e catalogo tematico.” Tesi di dottorato, Università degli Studi di Padova, 2009-2010. Capri, Antonio. Giuseppe Tartini. Milano: Garzanti, 1945.

Cartier, Jean Baptiste. L'art du violon, ou Collection choisie dans les sonates des Ecoles Italienne, Françoise et Allemande, précédée d'un abrégé de principes pour cet intrument; dédié au Conservatoire de Musique. Paris: Decombe, [1798].

Charlton, David. Opera in the Age of Rousseau. Music, Confrontation, Realism. Cambridge: Cambridge University Press, 2013.

Choron, Alexandre-Etienne. Dictionnaire historique des musiciens. Paris: ValadeLenorman, 1810.

D’Alay, Mauro. Cantate a voce sola e Suonate a violino solo col basso. London: [s. e.], 1728. D’Aquin de Château-Lyon, Pierre Louis. Siècle littéraire de Louis XV ou lettres sur les hommes célèbres. Amsterdam: Duchesne, 1754. 
Desmet, Marc. “Tartini e la Francia.” In Tartini »Maestro« narodov / Tartini "Maestro delle Nazioni”, a cura di Metoda Kokole, 89-100. Ljubljana: ZRC SAZU, Založba ZRC, 2002.

Devriès, Anik e Lesure, François. Dictionnaire des éditeurs de musique français. Genève: Minkoff, 1979.

Devriès-Lesure, Anik. Edition et commerce de la musique gravée à Paris dans la première moitié du XVIII siècle: les Boivin, les Leclerc. Genève: Minkoff, 1976.

___ . "Paris et la dissémination d'éditions musicales entre 1700 et 1830." Revue de Musicologie 84, n. 2 (1998): 293-298.

___ L L Lédition musicale dans la presse parisienne au XVIII siècle. Catalogue des annonces. Paris: CNRS Éditions, 2005.

Duckles, Vincent Harris e Elmer, Minnie Agnes. Thematic Catalog of a Manuscript Collection of Eighteenth-century Italian Instrumental Music in the University of California, Berkeley, Music Library. Berkeley: University of California Press, 1963.

Durante, Sergio. "Tartini and his texts." In Sergio Durante, Studi su Mozart e il Settecento / Studies on Mozart and the 18 ${ }^{\text {th }}$ Century, 167-207. Lucca: LIM, 2007.

Felici, Candida. “Non suona, canta su '1 violino': From Aesthetics to Compositional and Performance Practice in Tartini's Instrumental Music.” Ad Parnassum 11, n. 22 (2013): 127-141.

Grimm, Friedrich Melchior von. Lettre de M. Grimm sur Omphale. [Paris: s. e.], 1752. . "Lettre de M. Grimm à l'Abbé Raynal sur les remarques au sujet de sa lettre d'Omphale." Mercure de France maggio (1752): 187-192.

Hogwood, Christopher e McVeigh, Simon. “Giardini, Felice.” New Grove Music Online. Oxford Music Online. Accesso il 10 giugno 2014. (http://www.oxfordmusiconline.com.). La querelle des bouffons. A cura di Denise Launay. Genève: Minkoff, 1973.

Les Affiches de Paris.

Lesure, François. Bibliographie des éditions musicales publiées par Estienne Roger et Michel-Charles le Cène (Amsterdam, 1696-1743). Paris: Heugel et C ${ }^{\mathrm{ie}}, 1969$.

Mercure de France.

Pagin, André Noël. Sonates a violon seul et basse-continue dediées a Monseigneur le Prince de Grimberghen. P. ${ }^{\text {er }}$ Euvre. Gravée par Le S. ${ }^{r}$ Hue. Paris: l'Auteur-BoivinLe Clerc, [1748].

Pavanello, Agnese. "Il Trillo del diavolo di Giuseppe Tartini nell'edizione di Jean Baptiste Cartier.” Recercare 11 (1999): 265-279.

___ . 'From the 'Devil's Trill' to 19th-Century Bravura Studies: The Presence and Reception of Tartini's Music in Early 19th-Century France.” Ad Parnassum 11, n. 22 (2013): 99-111.

Rasch, Rudolph, ed. Music Publishing in Europe 1600-1900. Berlin: Berliner Wissenschafts Verlag, 2007.

- The Music Publishing House of Estienne Roger and Michel-Charles le Cène 1696-1743. My Work on the Internet, Volume Four, Catalogue Taglietti-Trios. (http:// www.let.uu.nl/ Rudolf.Rasch/personal/Roger/Catalogue-Taglietti-Trios.pdf.)

" "Publishers and Publishers." In Music Publishing in Europe 1600-1900, ed. by Rudolph Rasch, 194-195. Berlin: Berliner Wissenschafts Verlag, 2007. 
[Rousseau, Jean-Jacques]. Lettre à M. Grimm, au sujet des remarques ajoutées à sa lettre sur Omphale. [Paris: s. e.], 1752.

[s. n.]. Remarques au sujet de la Lettre de M. Grimm sur Omphale. Paris: [s. e.], 1752.

Tartini, Giuseppe. Sei Concerti a Cinque è Sei Stromenti [...] Del Signor, Giuseppe Tartini, di Padoua Opera Prima Libro Primo. Amsterdam: Le Cène, [s. a.].

___ - Sei Concerti a Cinque Stromenti [... O Opera Prima Libro Secondo. Amsterdam: Le Cène, [s. a.].

___ - Sei Concerti a Cinque Stromenti [...] delli Signori Giuseppe Tartini e Gasparo Visconti Opera Prima Libro Terzo. Amsterdam: Le Cène, [s. a.].

___ VI Sonate a Violino e Violone o Cimbalo Del Signor Giuseppe Tartini di Padoua opera prima. Amsterdam: Witvogel, [s. a.].

___ - Sonate a Violino e Violoncello o Cimbalo Dedicate a Sua Eccellenza il Signor Girolamo Ascanio Giustiniani da Giuseppe Tartini Opera Prima. Amsterdam: Le Cène, [1734].

___ - VI Sonate a Violino e Violoncello o Cimbalo Opera Seconda di Giuseppe Tartini di Padoa. Amsterdam: Le Cène, [1743].

___ Sonate a violino e basso dedicate al S. ${ }^{r}$ Guglielmo Fegeri di Giuseppe Tartini opera seconda. Roma: Cleton, 1745.

____ Nouvelle étude pour le violon ou Manière de varier et orner un pièce dans le gout du Cantabile Italien. par M. ${ }^{r}$ Pétronio Pinelli virtuoso Romano. Augmenté d'une Gavotte de Corelli, travaillez et doublez Par M. ${ }^{r}$ Giuseppe Tartini. Paris: Hue, [s. a.].

___ Sonates a violon seul avec la Basse Continue [... . Euvre IV.e. Paris: Hue, [ca. 1747].

____. "Lettera del defonto Signor Giuseppe Tartini alla Signora Maddalena Lombardini." L'Europa Letteraria, tomo V, parte seconda (1770): $74 \mathrm{sgg}$.

___ _ "Lettre de Feu Tartini à Madeleine Lombardini." Journal de musique 2 (1771): $15-30$.

___ . Traité des agrémens de la musique [...] traduit par le Sigr P. Denis. Paris: [s. e.], 1771 [ed. moderna a cura di Erwin R. Jacobi. Celle: Moeck, 1961].

____ L L L raccolta di sonate autografe per violino. Padova: Edizioni dell'Accademia Tartiniana, 1976. 


\section{ŠIRJENJE GLASBE GIUSEPPA TARTINIJA V FRANCIJI IZDAJE SONAT ZA VIOLINO IN BASSO CONTINUO IZ 18. STOLETJA}

\section{Povzetek}

Članek analizira francoske edicije 18. stoletja sonat za violino in bas Giuseppa Tartinija v kontekstu razširjenosti glasbe istrskega skladatelja v Franciji. Preko pregleda najav v sočasnih revijah in katalogov založnikov je možno določanje datumov izida zbirk sonat op. 4 in op. 9 v obdobju med 1747 in 1750. Primerjanje z rokopisno izdajo sonat in s katalogom, ki ga je pripravil ob koncu 18. stoletja Tartinijev učenec in naslednik Giulio Meneghini, je omogočilo ocenjevanje pristnosti teh natisov, med katerimi sta op. 6 in op. 7 ediciji, ki imata največ stičnih točk z najpomembnejšimi rokopisnimi zbirkami Tartinijevega sonatnega repertoarja, čeprav ni nobenih pričevanj o stikih med Tartinijem in francoskimi založniki. Slednji, ki so verjetno dobili omenjene sonate od »učitelja narodov«, niso poskrbeli za Tartinijevo dovoljenje, verjetno tudi zato, ker ni bilo nobenega zakona, ki bi branil avtorja. Podobno se je zgodilo že z nizozemskimi založniki Witvogel za op. 1 in Le Cène za op. 2. Preko analize izvedb Tartinijevih skladb na Concerts spirituels, predvsem tistih enega najbolj priljubljenih učencev Andréja Noëla Pagina v istih letih, ko so natisnili zbirke sonat, je bilo mogoče opisati francoski uspeh Tartinija, uspeh, ki je doživel svoj višek v polovici stoletja in ki se ga je tangentno dotaknila tudi querelle des bouffons. Pagin je tudi vezan na natis Tartinijevih sonat, ker sta op. 4 in op. 5 posvečena njemu in ker se pojavi kot urednik revidirane edicije op. 7. Čeprav v polovici osemdesetih Tartinijev kompozicijski stil in njegova izvedbena praksa nista bila več aktualna (kot priča znana kritika izvedbe Maddalene Lombardini Sirmen leta 1785), je verjetno Tartinijev pedagoški sloves doživljal v Franciji veliko odobravanja, če je bilo ob koncu stoletja, ko je Napoleon dal zaseči veliko umetniških del, knjig in rokopisov iz različnih italijanskih krajev zato, da bi okrepil francosko dediščino, med temi veliko Tartinijevih rokopisov, ki so obogatili zapuščino novonastalega pariškega Conservatoire de Musique. 